\title{
Porque estudar a morte? - Uma análise de oito anos
}

Mariana Tudela, ${ }^{*}$ Joana Melo Cabrita, ${ }^{* *}$ Maria José Ribas***

\section{RESUMO}

Objectivo: Analisar as causas de morte numa lista de utentes e factores associados.

Tipo de Estudo: Descritivo

Local: USF Horizonte, Matosinhos.

População: Utentes inscritos na lista de uma médica de família entre 2000 e 2007.

Métodos: Estudaram-se os óbitos ocorridos na lista de uma médica de família em 8 anos. Os dados foram colhidos pela consulta do processo clínico e programas informáticos $\mathrm{SAM}^{\circledR}, \mathrm{SAPE}{ }^{\circledR}$ e Piloto32 ${ }^{\circledR}$. Avaliaram-se variáveis sociodemográficas e relacionadas com o óbito. Realizou-se uma análise descritiva através do software Excel.

Resultados: Em oito anos, há registo de terem morrido 106 utentes, correspondendo a uma taxa de mortalidade de 8,7\%o utentes/ano. Dos óbitos registados, $62,2 \%$ dos doentes eram homens e a média de idades era de 71,6 anos. A maioria era reformada (79,2\%), casada (50,9\%) e apresentava limitação para actividades da vida diária (58,4\%). Dos doentes falecidos, 53,1\% morreram no hospital, $40,8 \%$ no domicílio e $7,6 \%$ em local desconhecido. O cuidador foi identificado em $90,6 \%$ dos casos, sendo um familiar em 55,7\%. A causa de morte era desconhecida em $8,5 \%$ dos óbitos e $47,4 \%$ resultaram de neoplasias.

Conclusões: Os resultados revelam fragilidades na notificação e registo de informação sobre a morte dos doentes. Os dados demográficos foram semelhantes a outros estudos, bem como a distribuição dos cuidadores entre familiares/não familiares. A idade média de morte foi inferior à encontrada em Barcelona e Israel. A maior diferença entre os resultados apresentados e a literatura residiu nas causas de morte que, nesta lista, não são as doenças cardiovasculares.

Palavras-chave: Causas de Mortalidade; Registo dos Óbitos; Cuidados de Saúde Primários.

«By tellingyourself the truth, you allowyourself to heal.» David Kuhl, in «Facing death: embracing live».

\section{INTRODUÇÃO}

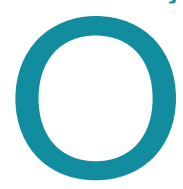

s médicos de família são detentores de informação importante sobre os seus doentes. Acompanham-nos ao longo do seu ciclo de vida, incluindo por vezes o momento da morte, dando também apoio aos familiares na fase de luto. Os registos clínicos efectuados ao longo das suas vidas contêm a história natural da maioria das doenças agudas e crónicas dos doentes que recorrem aos centros de saúde e podem dar informações preciosas sobre as causas e circunstâncias da morte. ${ }^{1}$ E que fazem dessa informação?

\footnotetext{
* Médica de Família, USF Porta do Sol, ACES/ULS de Matosinhos.

**Médica de Família, UCSP da Senhora da Hora, ACES/ULS de Matosinhos.

***Médica de Família, USF Garcia de Orta, ACES Porto Ocidental.
}

À data de realização deste trabalho, não existiam em Portugal estudos publicados sobre as causas de morte em Cuidados de Saúde Primários (CSP) ou reflexões sobre o interesse de as estudar. No entanto, um registo adequado dos óbitos permite avaliar as deficiências e monitorizar tendências na saúde, trabalhando as competências e recursos disponíveis, estabelecendo prioridades e implementando medidas correctoras, como estratégias mais apropriadas de cuidados ao domicílio. Desenvolve-se assim uma atitude pró-activa da equipa de saúde, com melhoria da qualidade dos cuidados e da satisfação dos profissionais da equipa. ${ }^{2-6}$

Os relatórios de actividades anuais em CSP dão pouca importância ao número de utentes que morrem e às suas causas básicas de morte. Além de que nem sempre é rotina, no nosso país e segundo a literatura, o envio de informação sobre a morte dos doentes ao médico de família, quando o processo final de vida não ocorre no domicílio e este não é acompanhado pelo próprio médico. ${ }^{3,7}$

Um exercício de reflexão contínua sobre este tema per- 
mite traçar estratégias de futuro, oferecendo uma maior compreensão e conhecimento na prática clínica, uma perspectiva diferente da interacção médico-doente e das qualidades do profissional, que adquire também maior insight pessoal. Os médicos de família são detentores de toda esta informação, frequentemente guardada para sempre nos registos clínicos sem ser objecto de qualquer reflexão individual ou da equipa.

O objectivo deste estudo foi analisar as causas de morte da lista de utentes de um médico de família e factores associados.

\section{MÉTODOS}

Realizou-se um estudo descritivo na Unidade de Saúde Familiar (USF) Horizonte em Matosinhos entre Dezembro de 2007 e Junho de 2008.

A população em estudo incluiu os utentes inscritos na lista de um médico de família, entre 01 de Janeiro de 2000 e 31 de Dezembro de 2007.

Para a determinação da taxa de mortalidade foram incluídos todos os indivíduos em observação ao longo dos 8 anos. Na análise das causas de morte foram incluídos todos os utentes falecidos no período dos 8 anos em estudo.

Os dados foram colhidos através da consulta do processo clínico e de programas informáticos de registo de dados (Piloto $32^{\circledR}$, Sistema de Apoio ao Médico ${ }^{\circledR}$ eSistema de Apoio à Enfermagem ${ }^{\oplus}$.

Avaliaram-se as seguintes variáveis sociodemográficas: género, idade (variável contínua), estado civil (solteiro, casado, viúvo e divorciado/separado) e escolaridade (analfabeto, sabe ler e escrever, ensino primário completo, ensino preparatório completo, 9. ${ }^{\circ}$ ano, $12 .^{\circ}$ ano, ensino superior e desconhecido).

As variáveis relacionadas com o óbito foram: local do óbito (domicílio, hospital, lar, via pública e desconhecido), causa do óbito, comorbilidades, grau de incapacidade do indivíduo na altura do óbito (Índice Karnovsky), cuidador imediatamente antes do óbito (cônjuge, filha/o, outro familiar, não familiar, sem cuidador, não aplicável e desconhecido) e fonte de informação do óbito (cônjuge, filha/o, outro familiar, não familiar, funerária, sistema informático, desconhecido).

As causas de morte foram estratificadas por género.

Os dados recolhidos foram codificados, registados em base de dados informática e analisados com o software Microsoft Office Excel $^{\circledR}$, em estatística descritiva.

\section{RESULTADOS}

Nos oito anos em estudo morreram 106 utentes da lista, correspondendo a uma média de 13 indivíduos por ano. Nesse período de tempo foi calculada uma população em observação de 12176 pessoas. Desconhecia-se a data de óbito de dois indivíduos. A taxa de mortalidade calculada foi de 8,7\%o utentes / ano (Quadro I).

Dos utentes que faleceram, $62,3 \%$ eram homens, com uma taxa de mortalidade no sexo masculino de $11,4 \%$ o por ano, e $37,7 \%$ eram mulheres, calculando-se uma taxa de mortalidade no sexo feminino de $5,5 \%$ o por ano.

De acordo com o Quadro II, a média de idades foi de 71,6 anos, variando entre 5 e 90 anos de idade. Os óbitos distribuíram-se por quase todas as faixas etárias, mas a maioria dos utentes encontrava-se entre os 71 e os 90 anos. A maioria dos óbitos ocorreu em utentes reformados $(79,2 \%)$, casados $(50,9 \%)$ e que apresentavam um nível de escolaridade baixo. Na altura da morte $58,4 \%$ dos doentes apresentavam limitação para actividades da vida diária, avaliada pela Escala de Karnovsky. Desconhecia-se o grau de capacidade funcional em $26,4 \%$ dos casos. Dos casos conhecidos, 79,5\% apresentavam limitações funcionais, valor que foi ligeiramente inferior ao encontrado em Israel. ${ }^{3} \mathrm{Em}$ 90,6\% dos casos foi identificado o cuidador do utente falecido, tratando-se do cônjuge ou filho(a) em $55,7 \%$.

Foi possível identificar a fonte da notícia do óbito ao médico de família em $76,4 \%$ dos casos, tratando-se de um fa-

QUADRO I. Taxa de mortalidade anual da lista de utentes e taxa de mortalidade no período total de estudo

\begin{tabular}{|lccc} 
Ano & $\begin{array}{c}\text { N. }{ }^{\circ} \text { de utentes } \\
\text { da lista (em 31 } \\
\text { de Dezembro) }\end{array}$ & N. ${ }^{\circ}$ de óbitos & $\begin{array}{c}\text { Taxa de } \\
\text { mortalidade/ } \\
/ 1000 \text { utentes }\end{array}$ \\
\hline 2000 & 1402 & 17 & 12,1 \\
2001 & 1460 & 14 & 9,6 \\
2002 & 1489 & 14 & 9,4 \\
2003 & 1520 & 8 & 5,3 \\
2004 & 1548 & 8 & 5,2 \\
2005 & 1566 & 20 & 12,8 \\
2006 & 1586 & 14 & 8,8 \\
2007 & 1605 & 9 & 5,6 \\
Desconhecido & - & 2 & - \\
\hline Total & 12176 & 106 & 8,7 \\
\hline
\end{tabular}


miliar em $57,6 \%$ dos casos. O local de morte era conhecido em $92,4 \%$ dos óbitos, sendo que destes $53,1 \%$ morreram no hospital e 40,8\% no domicílio (Quadro III).

Relativamente à causa de morte, esta foi identificada em $91,5 \%$ dos óbitos, dos quais $47,4 \%$ resultaram de neoplasias, $33 \%$ de doenças cardiovasculares e $7,2 \%$ de doenças respiratórias (Figura 1). Ocorreram quatro casos de mortes não naturais.

As causas de morte estratificadas por género encontram-se no Quadro IV. As causas de morte mais frequentes nos homens foram: neoplasia, acidente vascular cerebral (AVC),

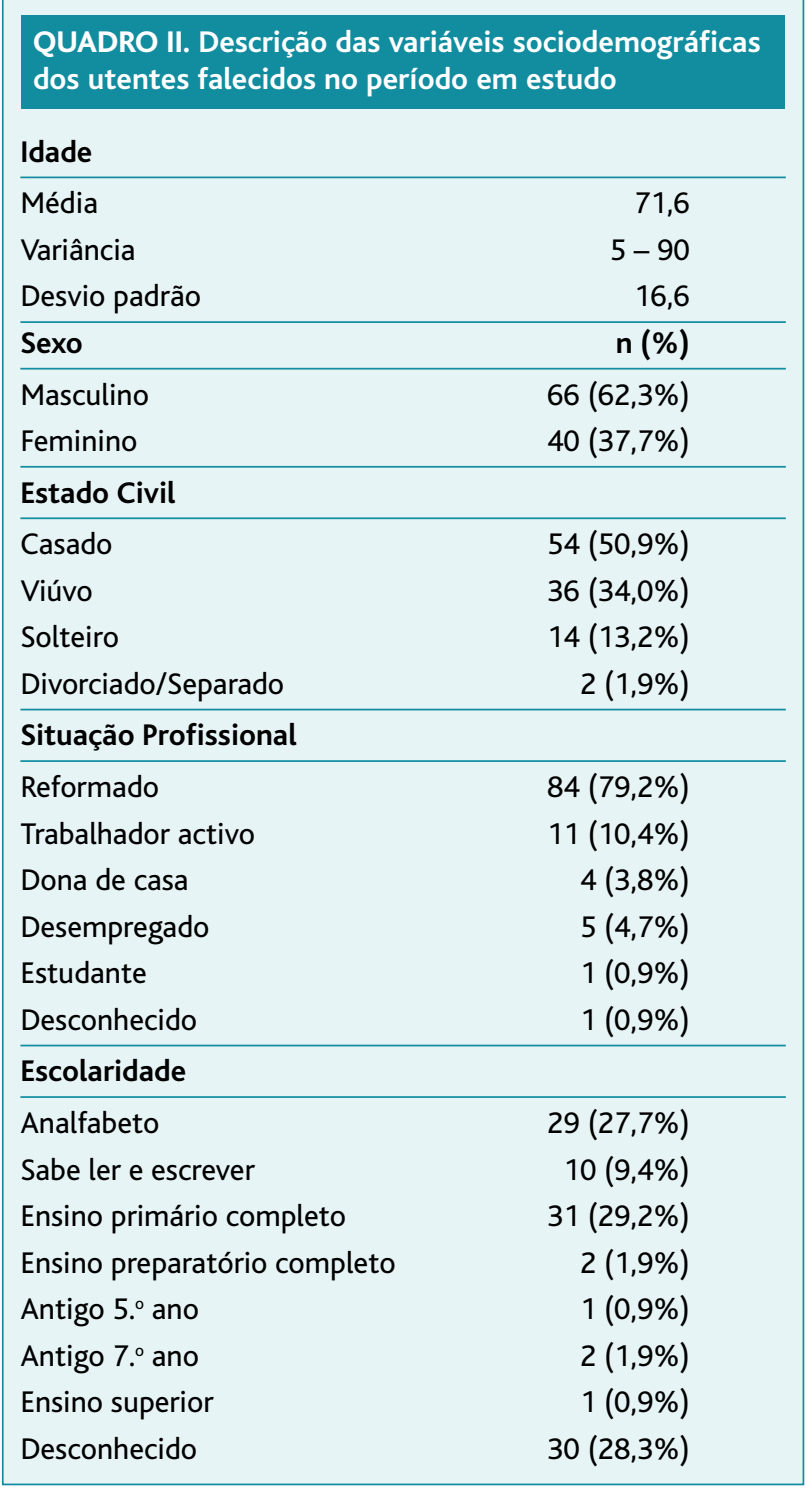

Legenda - n: número de indivíduos; \%: percentagem pneumonia e enfarte agudo do miocárdio e as causas de morte mais frequentes nas mulheres foram: neoplasia, AVC e insuficiência cardíaca congestiva.

No Quadro V são descritos os tipos de neoplasia que foram causa de morte, sendo a mais comum a neoplasia pulmonar, perfazendo $21,7 \%$ dos casos, seguida de neoplasia

\begin{tabular}{|c|c|}
\hline \multicolumn{2}{|c|}{$\begin{array}{l}\text { QUADRO III. Descrição das variáveis relacionadas com } \\
\text { o óbito }\end{array}$} \\
\hline Cuidador & $\mathrm{n}(\%)$ \\
\hline Cônjuge & $30(28,3 \%)$ \\
\hline Filho/a & $29(27,4 \%$ \\
\hline Outro familiar & $14(13,2 \%)$ \\
\hline Não familiar & $7(6,6 \%)$ \\
\hline Sem cuidador & $7(6,6 \%)$ \\
\hline Não aplicável & $15(14,2 \%)$ \\
\hline Desconhecido & $10(9,4 \%)$ \\
\hline \multicolumn{2}{|c|}{ Grau de Capacidade Funcional (Escala de Karnovsky) } \\
\hline $80-100 \%$ & $16(15,1 \%)$ \\
\hline $60-70 \%$ & $8(7,6 \%)$ \\
\hline $40-50 \%$ & $16(15,1 \%)$ \\
\hline $10-30 \%$ & $38(35,8 \%)$ \\
\hline Desconhecido & $28(26,4 \%)$ \\
\hline \multicolumn{2}{|l|}{ Fonte da notícia de morte } \\
\hline Cônjuge & $30(28,3 \%)$ \\
\hline Filho/a & $16(15,1 \%)$ \\
\hline Outro familiar & $15(14,2 \%)$ \\
\hline Não familiar & $5(4,7 \%)$ \\
\hline Funerária & $15(14,2 \%)$ \\
\hline Sistema informático & $5(4,7 \%)$ \\
\hline Desconhecido & $20(18,9 \%)$ \\
\hline \multicolumn{2}{|l|}{ Local da morte } \\
\hline Hospital & $52(49,1 \%)$ \\
\hline Domicílio & $40(37,7 \%)$ \\
\hline Lar & $5(4,7 \%)$ \\
\hline Via pública & $1(0,9 \%)$ \\
\hline Desconhecido & $8(7,6 \%)$ \\
\hline \multicolumn{2}{|l|}{ Comorbilidades mais frequentes } \\
\hline Hipertensão arterial & $51(48,1 \%)$ \\
\hline Diabetes mellitus tipo 2 & $27(25,5 \%)$ \\
\hline Insuficiência cardíaca congestiva & $22(20,8 \%)$ \\
\hline Doença cérebro-vascular & $12(11,3 \%)$ \\
\hline Fibrilhação auricular & $12(11,3 \%)$ \\
\hline
\end{tabular}


gástrica $(15,2 \%)$ e do cancro colo-rectal (13,0\%). Verificou-se uma grande variabilidade de neoplasias que conduziram ao óbito (dezoito tipos diferentes), sendo que apenas uma era passível de rastreio.

Foram também estudadas as comorbilidades apresentadas pelos utentes falecidos, sendo a hipertensão arterial a mais prevalente $(48,1 \%$ dos casos), seguida da diabetes mellitus (25,5\%) e insuficiência cardíaca congestiva $(20,8 \%)$ (Quadro III).

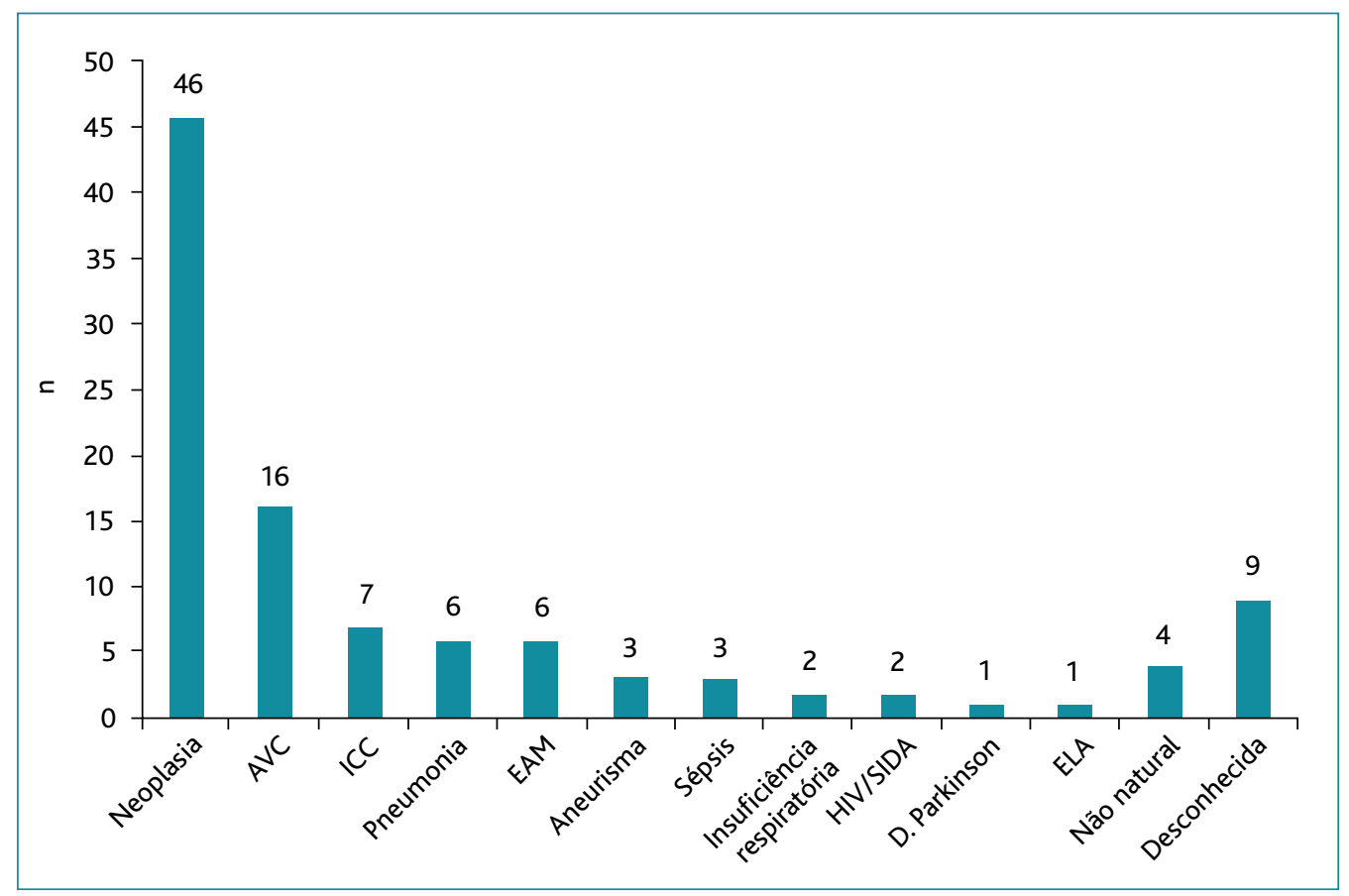

Figura 1. Distribuição das causas de morte por número absoluto.

\section{DISCUSSÃO}

Os resultados revelam fragilidades importantes no sistema de notificação e registo de informação sobre a morte dos doentes, o que nos deve fazer rever procedimentos individuais e colectivos.

A taxa de mortalidade obtida ( $8,7 \%$ o) foi um pouco superior à encontrada em Matosinhos em $2005(7,4 \% 0),{ }^{7}$ mas inferior ao valor nacional no mesmo ano $\left(10,1 \%\right.$ o). ${ }^{8}$ Comparando com outros estudos, a taxa de mortalidade anual não ficou muito distante da determinada em Espanha e em Israel, encontrando-se num valor intermédio relativamente aos restantes estudos (Quadro VI). ${ }^{2-4,6,9}$

Neste estudo a média de idades em que ocorreu o óbito foi ligeiramente inferior à encontrada na literatura (Israel 78,2 e Barcelona 73,6 anos), o que pode ser explicado pelo facto de ter sucedido um óbito numa criança., ${ }^{3,10}$

Quanto às causas de morte, obteve-se uma proporção bastante superior de doenças neoplásicas, relativamente

\begin{tabular}{lrrr}
\hline QUADRO IV. Estratificação das causas de morte por género \\
\hline Causa de Morte & \multicolumn{1}{l}{ Homens } & \multicolumn{1}{l}{ Mulheres } & \multicolumn{1}{l}{ Total } \\
\hline Neoplasia & $32(69,6 \%)$ & $14(30,4 \%)$ & $46(43,4 \%)$ \\
Acidente vascular cerebral & $9(56,3 \%)$ & $7(43,7 \%)$ & $16(15,1 \%)$ \\
Insuficiência cardíaca congestiva & $1(14,3 \%)$ & $6(85,7 \%)$ & $7(6,6 \%)$ \\
Pneumonia & $5(83,3 \%)$ & $1(16,7 \%)$ & $6(5,7 \%)$ \\
Enfarte agudo do miocárdio & $5(83,7 \%)$ & $1(16,7 \%)$ & $6(5,7 \%)$ \\
Aneurisma & $0(0,0 \%)$ & $3(100 \%)$ & $3(2,8 \%)$ \\
Sépsis & $1(33,3 \%)$ & $2(66,7 \%)$ & $3(2,8 \%)$ \\
Insuficiência respiratória & $2(100 \%)$ & $0(0,0 \%)$ & $2(1,9 \%)$ \\
HIV / SIDA & $2(100 \%)$ & $0(0,0 \%)$ & $2(1,9 \%)$ \\
Doença de Parkinson & $0(0,0 \%)$ & $1(100 \%)$ & $1(0,9 \%)$ \\
Esclerose Lateral Amiotrófica & $0(0,0 \%)$ & $1(100 \%)$ & $1(0,9 \%)$ \\
Não natural & $3(75,0 \%)$ & $1(25,0 \%)$ & $4(3,8 \%)$ \\
Desconhecida & $6(66,7 \%)$ & $3(33,3 \%)$ & $9(8,4 \%)$ \\
\hline Total & $66(62,3 \%)$ & $40(37,7 \%)$ & $106(100 \%)$ \\
& & &
\end{tabular}

aos valores nacionais, e uma proporção aproximada de mortes por doenças CV e por outras causas (Quadro VII). ${ }^{8}$

Comparando com os estudos internacionais, a proporção de neoplasias obtida mantém-se muito superior. ${ }^{2-4,6,7,10,11}$ Mesmo nos estudos em que esta proporção foi superior à 


\begin{tabular}{|c|c|}
\hline Tipo de Neoplasia & $N(\%)$ \\
\hline Cancro do pulmão & $10(21,7 \%)$ \\
\hline Cancro gástrico & $7(15,2 \%)$ \\
\hline Cancro colo-rectal & $6(13,0 \%)$ \\
\hline Cancro do pâncreas & $4(8,7 \%)$ \\
\hline Cancro da bexiga & $2(4,3 \%)$ \\
\hline Cancro da próstata & $2(4,3 \%)$ \\
\hline Cancro da via biliar ampola de vater & $2(4,3 \%)$ \\
\hline Leucemia/Linfoma & $2(4,3 \%)$ \\
\hline Cancro do fígado & $1(2,2 \%)$ \\
\hline Cancro do ovário & $1(2,2 \%)$ \\
\hline Cancro da parótida & $1(2,2 \%)$ \\
\hline Cancro renal & $1(2,2 \%)$ \\
\hline Cancro do útero & $1(2,2 \%)$ \\
\hline Cancro da vesícula biliar & $1(2,2 \%)$ \\
\hline Mieloma múltiplo & $1(2,2 \%)$ \\
\hline Sarcoma & $1(2,2 \%)$ \\
\hline Cancro da amígdala & $1(2,2 \%)$ \\
\hline Cancro do cavum & $1(2,2 \%)$ \\
\hline Glioblastoma & $1(2,2 \%)$ \\
\hline
\end{tabular}

das doenças CV, como em Espanha ${ }^{10} \mathrm{e}$ em Inglaterra ${ }^{4}$, a ordem de valores foi bastante menor do que a encontrada pelas autoras (Quadro VIII).

Neste estudo os doentes morreram mais no hospital, tal como verificado na maioria dos dados internacionais (Quadro IX) ${ }^{2,3,11}$ No entanto, a proporção relativa de doentes que morreram em casa foi maior do que nos mesmos.

Em Portugal falta um sistema de notificação oficial ao médico de família e a outros médicos cuidadores do doente. Esta noção vai de encontro aos resultados obtidos, em que a fonte da notícia do óbito foi predominantemente de tipo informal, constituída por familiares.

O estudo apresenta algumas limitações, dado que se baseia nos registos clínicos, por vezes colmatados com a memória do médico. Além disso, verificou-se que a informação sobre a circunstância de morte dos doentes era frequentemente insuficiente.

De referir ainda a pequena dimensão da população em estudo, na qual poderia ter sido incluída toda a população da USF ou de outras Unidades de Saúde da mesma localidade, por exemplo, de forma a tornar o estudo mais representativo. Os Quadros VI eVIII mostram como alguns estudos comparativos analisam populações médias muito superiores.

Os pontos fortes relacionam-se com o facto do estudo ser realizado em CSP, de se ter estudado a toda a lista de utentes e daglobalidade dos resultados não diferir muito dos encontrados na literatura.

\section{QUADRO VI. Comparação entre a taxa de mortalidade obtida neste estudo com a de outros estudos encontrados}

\section{Estudo}

População média N $\quad{ }^{\circ}$ de óbitos

Taxa de Mortalidade (\%o/ano)

Estudo actual, Matosinhos 2000/07 1577 160 8,7 Espanha 1987/95 22928 $-$ 7,1 Israel 1998/033 $\sim 6000$ 233 Holanda 1998/00 52471 Inglaterra 1994/984 $\sim 13000$ 841 270 $3,4-14,7^{*}$ (n) Inglaterra 1992/96 11 30790 1263

Censo

$2,447,864$

Manchester: 2,7

Northwitch: 3,5

Legenda - * variou muito entre as listas de diferentes médicos de família; EUA: Estados Unidos da América; N.: número.

\section{MAS, ENTÃO, POR QUE ESTUDAR A MORTE?}

Antes de mais para fazer uma auto-avaliação: saber de que estão a morrer os nossos doentes e questionarmo-nos se haverá grandes variações no número de doentes que morrem e nas causas de mortalidade, em comparação com outras listas, com os valores nacionais e internacionais.

Estudar os óbitos permite ainda coordenar melhor os recursos entre a equipa deCSP e com os Cuidados de Saúde Hospitalares. Permite apoiar o luto dos familiares de forma mais adequada e pode sobretudo gerar um trabalho de reflexão pessoal e interpares, analisando o su- 
porte prestado aos doentes terminais e à família, aprendendo a lidar melhor com as emoções ou sentimentos de culpa associados à morte dos nossos doentes e fazer o nosso próprio luto dos doentes «perdidos».

Esta reflexão deve ainda contemplar a forma como são preenchidos os certificados de óbito, pela influência que estes registos têm nas políticas de saúde. Neste estudo, os doentes morreram mais de doenças neoplásicas do

QUADRO VII. Comparação entre as causas de morte encontradas neste estudo e os valores nacionais em 2005

\begin{tabular}{lrrrr} 
Causas de morte & \multicolumn{2}{c}{ Estudo actual 1999/07 } & \multicolumn{2}{c}{ Portugal 2005} \\
& N & \% & N & $\%$ \\
\hline Doenças do aparelho circulatório & 32 & 30,2 & 36570 & 34,0 \\
Tumores malignos & 46 & 43,4 & 22682 & 21,1 \\
Diabetes mellitus & 0 & 0,0 & 4569 & 4,3 \\
Doenças do aparelho respiratório & 8 & 7,5 & 11288 & 10,5 \\
Doenças do aparelho digestivo & 0 & 0,0 & 4525 & 4,3 \\
Outras causas & 16 & 15,1 & 23247 & 21,6 \\
Acidentes, envenenamento, violência & 4 & 3,8 & 4481 & 4,2 \\
\hline
\end{tabular}
que cardiovasculares. Será que este achado representa um desvio relativamente ao valor nacional, ou estarão as doenças CV a ser a sobre-notificadas na generalidade?

Tendo em conta que apenas uma das neoplasias encontradas nas causas de morte era passível de rastreio, o estudo sugere-nos que deveremos estar mais atentos a certos sinais de doença, revendo os procedimentos preventivos adoptados e os restantes cuidados prestados aos indivíduos e famílias, nomeadamente os cuidados continuados integrados.

Tendo-se verificado a inexistência de um sistema de informação ao Médico de Família - uma notícia de óbito - revela-se essencial a criação de um registo sistemático, formalizado e centralizado dos indivíduos que morrem, que permita chegar essa informação a todos os cuidadores de saúde. Como, por exemplo, o envio da cópia do certificado de óbito.

Entre as reflexões apresentadas ficam as seguintes sugestões, consideradas de especial relevância:

- Introduzir «o estudo das taxas e causas de morte» nos relatórios de actividades anuais da USF

- Criar uma notificação sistemática da morte, como acontece já com as notícias de nascimento - introduzir esta ideia nos Agrupamentos dos Centros de Saúde (ACES) e Unidades Locais de Saúde (ULS)

- Discutir o tema inter-pares, em reflexão individual e da equipa

- Detectar necessidades formativas nas áreas relacionadas com a morte (dor, doente terminal, comunicação, certi-
QUADRO VIII. Comparação entre a proporção de doenças cardiovasculares e de neoplasias encontrada neste estudo e as proporções encontradas nos estudos internacionais obtidos

$\begin{array}{ccc}\text { Estudo } & \begin{array}{c}\text { População } \\ \text { média }\end{array} & \begin{array}{c}\text { Doenças } \\ \text { cardiovasculares }\end{array} \\ & & \text { Neoplasias }\end{array}$

Estudo actual, Matosinhos 2000/07 1577 $30,2 \%$ $43,4 \%$ Espanha 1987/95 22928 $33,8 \%$ $32,1 \%$ Espanha 1993/98 ${ }^{10}$ 37306

$17,3 \%$ $21,3 \%$ Israel 1998/033 $\sim 6000$ $36,0 \%$ $21,0 \%$ Holanda 1998/00 7 52471 $41,3 \%$ $29,1 \%$ Inglaterra 1994/984 Inglaterra 1992/96 ${ }^{11}$ $\sim 13000$ $25,9 \%$ $28,8 \%$ 30790 $40,6 \%$ $27,8 \%$ Londres $1997 / 00^{2}$ $2545-4900$ $37,2 \%$ $24,4 \%$

\begin{tabular}{|c|c|c|c|}
\hline \multicolumn{4}{|c|}{$\begin{array}{l}\text { QUADRO IX. Comparação entre os locais de óbito } \\
\text { detectados neste estudo e os de outros estudos } \\
\text { encontrados }\end{array}$} \\
\hline Estudo & Hospital & Domicílio & Outros \\
\hline Estudo actual 1999/07 & $49,1 \%$ & $37,7 \%$ & $13,2 \%$ \\
\hline Londres $1997 / 00^{2}$ & $64,1 \%$ & $24,8 \%$ & $11,1 \%$ \\
\hline Israel 1998/03³ & $48,0 \%$ & $15,0 \%$ & $37,0 \%$ \\
\hline Inglaterra 1992/96 ${ }^{11}$ & $60,0 \%$ & $22,4 \%$ & $17,4 \%$ \\
\hline Holanda $1998 / 00^{7}$ & $34,6 \%$ & $40,1 \%$ & $25,3 \%$ \\
\hline País de Gales 1964/855 & $37,0 \%$ & $59,0 \%$ & $4,0 \%$ \\
\hline
\end{tabular}


ficação do óbito, etc.) e com o preenchimento do certificado de óbito.

Seria também interessante realizar um estudo idêntico numa população mais alargada, de uma USF ou de um ACES, que incluísse a estratificação das causas de morte em função da idade e/ou o estudo de variáveis agrupadas (ex. causa do óbito/local do óbito), permitindo tirar elações mais robustas.

Por que não estudar a morte... para melhor pensar a vida?

\section{REFERÊNCIAS BIBLIOGRÁFICAS}

1. Kochanek KD, Smith BL. Deaths: Preliminary data for 2002. Natl Vital Stat Rep 2004 Feb 11; 52 (13): 1-48.

2. Beaumont B, Hurwitz B. Is it possible and worth keeping track of deaths within general practice? Results of a 15 year observational study. Qual Saf Health Care 2003 Oct; 12 (5): 337-42.

3. Rosenberg R, Vinker S, Yaphe J, Nakar S. The role of periodic mortality case review sessions in a primary care teaching clinic. Isr Med Assoc J 2006 Jun; 8 (6): 373-7.

4. Webb R, Esmail A. An analysis of practice-level mortality data to inform a health needs assessment. Br J Gen Pract 2002 Apr; 52 (477): 296-9.

5. Hart JT, Humphreys C. Be your own coroner: an audit of 500 consecutive deaths in a general practice. Br Med J (Clin Res Ed) 1987 Apr 4; 294 (6576): 871-4.
6. Palacios Soler F, Ojanguren Alonso M, Vázquez Goñi R, Moreno-Iribas C,Abad Vicente F. Estudio de la mortalidad en una zona básica de salud urbana. Aten Primaria 2001 Jun 30; 28 (2): 120-5.

7. Unidade de Saúde Pública de Matosinhos. Diagnóstico de Situação do Concelho de Matosinhos 2005. Matosinhos: USP de Matosinhos; 2005.

8. Instituto Nacional de Estatística. Nomenclatura das Unidades Territoriais para Fins Estatísticos. $3^{a}$ rev. (NUTS III). Disponível em: http://www.ine.pt [acedido a 20/12/2008]

9. Oppewal F, Meyboom-de Jong B. Mortality in general practice: an analysis of 841 deaths during a two-year period in 17 Dutch practices. Eur J Gen Pract 2004 Mar; 10 (1): 13-7.

10. Albadejo Blanco C, Parés Ubach Q, Monje Hernández A, López López MI. Causas de mortalidad en un centro de atención primaria. Aten Primaria 2003 Feb 28; 31 (3): 205-7.

11. Holden J, O'Donnell S, Brindley J, Miles L. Analysis of 1263 deaths in four general practices. Br J Gen Pract 1998 Jul; 48 (432): 1409-12.

\section{CONFLITOS DE INTERESSE}

As autoras declaram não possuir qualquer tipo de conflitos de interesse.

\author{
ENDEREÇO PARA CORRESPONDÊNCIA \\ Mariana Tudela \\ Rua Alfredo Keil, 371 - 5. Esq. \\ 4150-049 Porto \\ E-mail:marianatudela@gmail.com
}

Recebido em 08/01/2009

Aceite para publicação em 30/11/2010

\section{ABSTRACT}

\section{WHY STUDY DEATH? - AN ANALYSIS OF DEATHS FROM A FAMILY PRACTICE OVER AN EIGHT-YEAR PERIOD}

Objectives: To analyze the causes of death and factors associated with the death of patients from one family practice.

Type of study: Descriptive

Location: USF Horizonte, Matosinhos, Portugal

Population: Patients registered with one family doctor between 2000 and 2007.

Methods: The data were abstracted from clinic records using the Piloto $32^{\circledR}$, SAM $^{\circledR}$ and SAPE ${ }^{\circledR}$ electronic medical record systems. Socio-demographic variables and factors related to the death of the patients were analyzed using an Excel electronic spreadsheet.

Results: Over an eight year period, 106 deaths were recorded giving a mortality rate of 8.7 deaths per 1000 patients per year. Of these, $62.2 \%$ of the patients were male. The median age was 71.6 years. Most were retired $(79.2 \%)$ and most were married (50.9\%) at the time of death. The place of death was known in $92.4 \%$ of cases. Of these, $53.1 \%$ died in hospital and $40.8 \%$ died at home. Limitation in activities of daily living (ADL) was recorded among $58.4 \%$ of patients before their death. A primary caregiver was identified in $90.6 \%$ of cases. The caregiver was a family member in $55.7 \%$ of cases. A cause of death was unknown in $8.5 \%$ of cases. Neoplasm was recorded as the cause of death in $47.7 \%$ of cases.

Discussion: These results reveal deficiencies in the clinical recording of information about the death of patients and in the reporting of deaths. The demographic characteristics of this population are similar to those found in other published studies of mortality in primary care from Spain and Israel. The distribution of care among family and non-family caregivers is similar. The major differences between this population and others lie in the causes of death. Neoplasm was the most common cause of death and not cardiovascular disease, as has been found in other studies.

Keywords: Cause of Death; Mortality Registration; Primary Care. 